\title{
Extending Representability on the Set of Intervals Endowed with Admissible Orders for the Construction of Interval-valued Fuzzy Operators
}

\author{
Lidiane da Silva $^{a}$ and Guilherme Schneider ${ }^{a}$ and Bruna Freitas ${ }^{b}$ \\ Adenauer Yamin ${ }^{a}$ and Helida Santos ${ }^{b}$ and Benjamín Bedregal ${ }^{c}$ and Renata Reiser $^{a}$ \\ ${ }^{a}$ Centre for Technological Development, Federal University of Pelotas, Pelotas-RS, Brazil \\ \{lcdsilva, gbschneider, reiser, adenauer\}@inf .ufpel.edu.br \\ ${ }^{b}$ Centro de Ciências Computacionais, Universidade Federal do Rio Grande, \\ Rio Grande-RS Brazil, \{bruna.freitas, helida\}@furg.br \\ ${ }^{c}$ Departamento de Informática e Matemática Aplicada, Universidade Federal do Rio Grande do Norte, \\ Natal-RN, Brazil, \{bedregal\}@dimap.ufrn.br
}

\begin{abstract}
In this work, we provide a study on the representability of interval-valued fuzzy connectives considering partial and admissible orders. Our approach considers those orders based on injective aggregation functions which allowed the construction of intervalvalued operators. An immediate result is the construction of the implication used in the Quantum Logic, known as QL-implication, that in our proposal is generalised for intervals, given by means of interval-valued $\mathrm{t}-$ (co)norms and interval-valued fuzzy negations.
\end{abstract}

Keywords: interval-valued fuzzy operators, interval-valued fuzzy logic, QLimplications, admissible orders.

\section{Introduction}

Interval-valued Fuzzy Logic (IvFL) [17], seen in the narrow sense, has, among other objectives, the goal of dealing with uncertainty not restricted to membership functions, but also considering their relevance. Thus, the notion of "precise number", representing a membership degree, is extended to an interval value carrying its uncertainty in the unit interval. According to R. Moore and W. Lodwick [11], IvFL may be thought as arising from the need of a more complete and inclusive logical model of uncertainty, expressing computations with real numbers and their interrelations in Scott and Moore topology. Such approach emphasises the synergism between interval analysis (as developed by R. E. Moore, see [9, 11]) and fuzzy set theory (as conceived by [16]). See, e.g., fuzzy arithmetic, interval arithmetic on alpha-cuts, the extension principle and interval representability of fuzzy connectives. Focused on the latter, we have considered the canonical representation to interpret the truth degree of a fuzzy implication related to conditional rule in inference systems based on fuzzy logic, modelling the (lack of) knowledge concerning the value of a variable. So, by the best interval representation of a fuzzy implication, the correct result of such variable also aggregates the optimality criteria [8]. In order to achieve our goals, we focus our study on the representability of various interval-valued fuzzy operators considering partial and total/admissible orders. This leads to the introduction of interval-valued $\mathrm{t}$-(co)norms, fuzzy negations, and implications, including the ones constructed by means of these operators, namely, the class of QL-implications that are extended for intervals considering partial and admissible orders.

This paper is organised as follows: The main concepts of interval representations of real functions w.r.t. fuzzy connectives are presented in Sect. 2. Sect. 3 discusses partial and admissible orders for the family of all interval-valued fuzzy values $L([0,1])$ and in Sects. 4 and 5 , we have the representability considering those orders. Interval-valued fuzzy connectives, some of their properties and main classes are studied, including examples. In Sect. 6 we find the Conclusion.

\section{Preliminaries}

The interval-valued fuzzy structures in $[3,4]$ are conceived based on the concepts as correctness and optimality, both performing inferences and analysing criteria in fuzzy algorithms. Thus, the study of the main classes of interval-valued fuzzy connectives is considered, analysing the properties related to representability w.r.t. fuzzy connectives.

Consider $L([0,1])=\{[x, y] \mid 0 \leqslant x \leqslant y \leqslant 1\}$ as the family of all interval-valued fuzzy values. The projections $l, r: L([0,1]) \rightarrow[0,1]$ are defined by $l\left(\left[x_{1}, x_{2}\right]\right)=x_{1}$ and $r\left(\left[x_{1}, x_{2}\right]\right)=x_{2}$, respectively. For $X \in L([0,1]), l(X)$ and $r(X)$ are also denoted by $\underline{X}$ and $\bar{X}$, respectively. For $\vec{X}=\left(X_{1}, \ldots, X_{n}\right) \in L([0,1])^{n}$ : 
(i) $l(\vec{X})=\left(\underline{X_{1}}, \ldots, \underline{X_{n}}\right)$ and $r(\vec{X})=\left(\overline{X_{1}}, \ldots, \overline{X_{n}}\right)$;

(ii) $*(\vec{X})=\left[*\left(\underline{X_{1}}, \ldots, X_{n}\right), *\left(\overline{X_{1}}, \ldots, \overline{X_{n}}\right)\right]$, when $* \in\{\vee, \wedge\}$.

Let $D(L([0,1]))=\{[x, y] \in L([0,1]) \mid x=y\}$ be the set of all degenerate intervals, denoting a degenerate element $[x, x]=\mathbf{x}$, for $x \in[0,1]$.

Let $\mathbb{F}: L([0,1])^{n} \rightarrow L([0,1])$ be an interval-valued function $(\operatorname{IvF})$. The functions $\mathbb{F}, \overline{\mathbb{F}}: L([0,1])^{n} \rightarrow[0,1]$ are, respectively, given by $\mathbb{E}\left(X_{1}, \ldots, X_{n}\right)=l\left(\mathbb{F}\left(X_{1}, \ldots, X_{n}\right)\right.$, $\overline{\mathbb{F}}\left(X_{1}, \ldots, X_{n}\right)=r\left(\mathbb{F}\left(X_{1}, \ldots, X_{n}\right)\right)$.

\section{Admissible orders on $L([0,1])$}

This section introduces an admissible order inspired on contributions given by $[10,14]$, and also $[5,18]$,

Firstly, consider the two partial orders on $L([0,1])$ :

O1 Inclusion order: $X \subseteq Y$ iff $\underline{X} \geqslant \underline{Y}$ and $\bar{X} \leqslant \bar{Y}$;

O2 Product (Usual) order: $X \leq Y$ iff $\underline{X} \leqslant \underline{Y}$ and $\bar{X} \leqslant \bar{Y}$.

The degenerate intervals $[0,0]=\mathbf{0},[1,1]=\mathbf{1} \in$ $\mathbb{D}(L([0,1]))$ are, respectively, the smallest and the greatest elements of the lattice $(L([0,1], \leq)$.

A linear order over $L([0,1])$ is a binary relation which is transitive, antisymmetric and total. Equivalently, a linear order is a partial order under which every pair of intervals in $L([0,1])$ is comparable.

Definition 1. [5] An order $\preceq_{L([0,1])}$ is called an admissible order on $L([0,1])$ if the following holds:

(i) $\preceq_{L([0,1])}$ is a linear order on $L([0,1])$, and

(ii) $\preceq_{L([0,1])}$ refines a partial order $\leq$, meaning that: $\forall X_{1}, X_{2} \in L([0,1]), X_{1} \leq X_{2} \Rightarrow X_{1} \preceq_{L([0,1])} X_{2}$.

According with Definition 1, the degenerate intervals $\mathbf{0}$ and $\mathbf{1}$ are also the smallest and greatest elements of any $\left\langle L([0,1]), \preceq_{L([0,1])}\right\rangle$, respectively [5].

Example 1. In [12], the admissible orders are given:

- The order of Xu and Yager:

$$
X \preceq_{X Y} Y \Leftrightarrow\left\{\begin{array}{l}
\underline{X}+\bar{X}<\underline{Y}+\bar{Y}, \text { or } \\
\underline{X}+\bar{X}=\underline{Y}+\bar{Y} \text { and } \bar{X}-\underline{X} \leqslant \bar{Y}-\underline{Y}
\end{array}\right.
$$

- The first lexicographical order:

$$
X \preceq_{\text {Lex } 1} Y \Leftrightarrow\left\{\begin{array}{l}
\underline{X}<\underline{Y} \text {, or } \\
\underline{X}=\underline{Y} \text { and } \bar{X} \leqslant \bar{Y}
\end{array}\right.
$$

- The second lexicographical order:

$$
X \preceq_{\text {Lex2 }} Y \Leftrightarrow\left\{\begin{array}{l}
\bar{X}<\bar{Y}, \text { or } \\
\bar{X}=\bar{Y} \text { and } \underline{X} \leqslant \underline{Y}
\end{array}\right.
$$

In the next, a method for generating admissible orders $\left\langle L([0,1]), \preceq_{A}\right\rangle$ by a injective function $A$ is presented.
Theorem 2. Let $A: L([0,1]) \rightarrow[0,1]$ be a function such that $A$ is increasing w.r.t. a partial $\leq$-order such that $A(\mathbf{0})=0$ and $A(\mathbf{1})=1$. The $\preceq_{A}$-relation on $L([0,1])$ defined by

$$
X \preceq_{A} Y \Leftrightarrow\left\{\begin{array}{l}
X=Y, \text { or } \\
A(X)<A(Y),
\end{array}\right.
$$

is an admissible order on $L([0,1])$, when $A$ is injective.

Proof. The relation $\preceq_{A}$ is reflexive and antisymmetric, immediately. Let $X, Y, Z \in L([0,1])$ such that $X \preceq_{A} Y$ and $Y \preceq_{A} Z$. Then, the following hold:

(i) In case $X=Y$ or $Y=Z$, we immediately have that $X \preceq_{A} Z$. If $X \preceq_{A} Y$ and $Y \preceq_{A} Z$, the next cases hold:

(ii) In case $X \neq Y$ and $Y \neq Z$ then $A(X)<A(Y)$ and $A(Y)<A(Z)$ and therefore $A(X)<A(Z)$. So, $X \preceq_{A} Z$. (iii) In case $X \neq Y$ and $Y=Z$ then $A(X)<A(Y)$ and $A(Y)=A(Z)$, meaning that $A(X)<A(Z)$. So, $X \preceq_{A} Z$. (iv) In case $X=Y$ and $Y \neq Z, A(X)=A(Y)$ and $A(Y)<A(Z)$, then $A(X)<A(Z)$. So, $X \preceq_{A} Z$.

Based on the above cases, we have a transitive $\left\langle L([0,1]), \preceq_{A}\right\rangle$-relation. Now, for each $X, Y \in L([0 ; 1])$, we have that cases:

(i) In case $A(X)<A(Y)$, it implies that $X \preceq_{A} Y$;

(ii) In case $A(Y)<A(X)$, it implies $Y \preceq_{A} X$; and

(iii) If $A(X)=A(Y)$, since $\mathrm{A}$ is an injective function, it results on $X=Y$. Then, we also have a total $\left\langle L([0,1]), \preceq_{A}\right\rangle$-relation. Therefore, we have a linear $\left\langle L([0,1]), \preceq_{A}\right\rangle$-order. In addition, from the fact that when $X \leq Y$, then $A(X) \neq A(Y)$, by the injectivity of A, we have that either $A(X)<A(Y)$ or $A(Y)<A(X)$. Thus, it shows that $\preceq_{A}$-order is linear. Finally, consider $X, Y \in L([0,1])$, such that $X \leq Y$. If $X=Y$ then, trivially, $X \preceq_{A} Y$. For a partial $\leq$-order, if $X<Y$ then $A(X)<A(Y)$, since $A$ is injective and increasing. Therefore, $X \preceq_{A} Y$ and so, the $\preceq_{A}$-order refines the usual $\leq$-order. Concluding, $\preceq_{A}$-order is an admissible order on $L([0,1])$.

In the next proposition, we define the $\preceq_{\mathbf{A}}$-order, which is based on a function A obtained by the infinite decimal expansion of the endpoints of an interval $X=$ $[\underline{X}, \bar{X}] \subseteq[0,1]$, which is indicated as follows:

$$
[\underline{X}, \bar{X}]=\left[0 \cdot \underline{X}^{[1]} \underline{X}^{[2]} \ldots, 0 . \bar{X}^{[1]} \bar{X}^{[2]} \ldots\right] .
$$

And, we consider that $1=0.99 \ldots$ and $0=0.00 \ldots$.

Definition 3. Function A: $L([0,1]) \rightarrow[0,1]$ given by

$$
\mathbf{A}(X)=\left\{\begin{array}{l}
0 . \underline{X}^{[1]} \bar{X}^{[1]} \underline{X}^{[2]} \bar{X}^{[2]} \ldots, \text { if } 0 \leq \underline{X} \leq \bar{X}<1 \\
0 . \underline{X}^{[1]} 9 \underline{X}^{[2]} 9 \ldots, \text { if } 0 \leq \underline{X} \leq \bar{X}=1 \\
1, \text { if } X=\mathbf{1} ;
\end{array}\right.
$$


is an increasing function w.r.t. the usual partial $\leq$-order on $L([0,1])$ satisfying the boundary conditions $\mathbf{A}(\mathbf{0})=$ 0 and $\mathbf{A}(\mathbf{1})=1$.

Proposition 4. The function $\mathbf{A}: L([0,1]) \rightarrow[0,1]$ given in Eq. (2) is an injective function such that $X \leq$ $Y \Rightarrow \mathbf{A}(X) \leq \mathbf{A}(Y)$, for the usual $\leq$-order.

Proof. Straightforward from [14, Proposition 1].

Corollary 1. The $\preceq_{\mathrm{A}}$-order relation given as

$$
X \preceq_{\mathbf{A}} Y \Leftrightarrow\left\{\begin{array}{l}
X=Y, \text { or } \\
\mathbf{A}(X)<\mathbf{A}(Y),
\end{array}\right.
$$

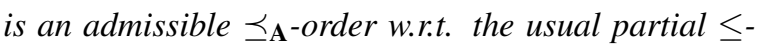
order on $L([0,1])$.

Proof. Straight from Theorem 2 and Prop. 4.

Example 2. Let $X=[0.15,0.88], Y=[0.16,0.86] \in$ $L([0,1])$. Then, we have that:

(i) $X \preceq_{\text {Lex } 1} Y, Y \preceq_{\text {Lex } 2} X$ and $Y \preceq_{X Y} X$;

(ii) $\mathbf{A}(X)=0.1858<0.1866=\mathbf{A}(Y)$, then $X \preceq_{\mathbf{A}} Y$.

Definition 5. Let $\mathbf{A}: L([0,1]) \rightarrow[0,1]$ given in Definition 3. For $x=0 . x^{[1]} x^{[2]} \ldots=\left(0 . x^{[i]}\right)_{i \in \mathbb{N}_{+}} \in[0,1]$ when $\mathbb{N}_{+}=\{1,2, \ldots\}$, the corresponding function $\mathbf{A}^{(-1)}:[0,1] \rightarrow L([0,1])$ is defined as follows

$$
\mathbf{A}^{(-1)}(x)=\left\{\begin{array}{l}
A^{(-1)}(x)=\mathbf{1} \text { if } x=1 \\
{\left[\left(0 . x^{[2 i-1]}\right)_{i \in \mathbb{N}_{+}},\left(0 . x^{[2 i]}\right)_{i \in \mathbb{N}_{+}}\right] \text {if }} \\
\quad\left(0 . x^{[2 i-1]}\right)_{i \in \mathbb{N}_{+}} \leq\left(0 . x^{[2 i]}\right)_{i \in \mathbb{N}_{+}} ; \text {and } \\
\inf \{X \in L([0,1]): A(X) \geq x\}=[x, x], \text { otherwise. }
\end{array}\right.
$$

Example 3. When $x=0.9819$ and $i \in \mathbb{N}_{2}$. then $A^{(-1)}(x)=[0.9819,0.9819]$ since $\left(0 . x^{[1]} x^{[3]}\right)=0.91 \geq$ $0.89=\left(0 . x^{[2]} x^{[4]}\right)$.

Lemma 1. The function $\mathbf{A}^{(-1)}:[0,1] \rightarrow L([0,1]) d e$ fined in Eq. (4) verifies the following conditions:

1. for each $X \in L([0,1])$, such that $\mathbf{A}(X) \neq 1$, $\mathbf{A}^{(-1)}(\mathbf{A}(X))=X$

2. for each $x \in[0,1], \mathbf{A}\left(\mathbf{A}^{(-1)}(x)\right) \leq x$;

3. if $x, y \in[0,1]$ and $x \leq y, \mathbf{A}^{(-1)}(x) \preceq_{\mathbf{A}} \mathbf{A}^{(-1)}(y)$;

4. for each $x \in[0,1], \mathbf{A}^{(-1)}(x)=\mathbf{0} \Leftrightarrow x=0$ and $\mathbf{A}^{(-1)}(x)=\mathbf{1} \Leftrightarrow x=1$.

Proof. Straightforward.

Example 4. If $x=0.85$ then 0.8 is not smaller than 0.5. So, $\mathbf{A}^{(-1)}(0.85)=[0.85,0.85]$. Thus, the corresponding infimum for the usual partial order $\mathbf{A}\left(\mathbf{A}^{(-1)}(0.85)\right)=0.8855>0.85$.

\section{Representability on $\langle L([0,1]), \leq\rangle$}

Let $\langle L([0,1])), \leq\rangle$ be the lattice of all interval-valued fuzzy values. In [6], an interval $X \in L([0,1])$ is said to be an interval representation of a real number $\alpha$ whenever $\alpha \in X$. By considering two interval representations $X$ and $Y$ of a real number $\alpha, X$ is said a better representation of $\alpha$ than $Y$ if $X \subseteq Y$.

Definition 6. $[15$, Section 1] A function $F: L([0,1])^{n} \rightarrow L([0,1])$ is an interval representation of a function $f:[0,1]^{n} \rightarrow[0,1]$ if, for each $\vec{X}=\left(X_{1}, \ldots, X_{n}\right) \in L([0,1])^{n}$ and $\vec{x} \in \vec{X}, f(\vec{x}) \in F(\vec{X})$.

In addition, an interval-valued function $F: L([0,1])^{n} \rightarrow$ $L([0,1])$ is a better interval representation of $f: L([0,1])^{n} \rightarrow L([0,1])$ than $G: L([0,1])^{n} \rightarrow L([0,1])$, denoted by $G \sqsubseteq F$, if for each $\vec{X} \in L([0,1])^{n}$, the inclusion $F(\vec{X}) \subseteq G(\vec{X})$ holds.

Definition 7. [15, Section 2] The best interval representation (canonical representation) of a real function $f:[0,1]^{n} \rightarrow[0,1]$, is the interval-valued function $\widehat{f}: L([0,1])^{n} \rightarrow L([0,1])$ defined by

$$
\widehat{f}(\vec{X})=[\inf \{f(\vec{x}) \mid \vec{x} \in \vec{X}\}, \sup \{f(\vec{x}) \mid \vec{x} \in \vec{X}\}] .
$$

The function $\widehat{f}$ is well defined and for any other interval representation $F$ of $f, F \sqsubseteq \widehat{f}$, providing a narrower interval than any other interval representation of $f$. Thus, $\widehat{f}$ has the optimality property of interval-valued algorithms mentioned by Hickey et al. [8], when it is seen as an algorithm to compute a real function $f$.

By [15, Sect. 2.2], for an Interval-valued function (IvF) $f: L([0,1])^{n} \rightarrow L([0,1])$, the following statements are equivalent: (i) $f$ is continuous; (ii) $\widehat{f}$ is Scott continuous; (iii) $\widehat{f}$ is Moore continuous.

So, when $f$ is continuous in the usual sense,

$$
\widehat{f}(\vec{X})=\{f(\vec{x}) \mid \vec{x} \in \vec{X}\}=f(\vec{X}), \forall \vec{X} \in L([0,1])^{n} .
$$

Moreover, taking $n \in \mathbb{N}_{2}, \mathbb{F}: L([0,1])^{n} \rightarrow L([0,1])$ is called an interval-valued decomposable function if there exist operations $F_{1}, F_{2}:[0,1]^{n} \rightarrow[0,1]$, such that for all $X_{1}, \ldots, X_{n} \in L([0,1])$

$$
\mathbb{F}\left(X_{1}, \ldots, X_{n}\right)=\left[F_{1}\left(\underline{X}_{1}, \ldots, \underline{X}_{n}\right), F_{2}\left(\bar{X}_{1}, \ldots, \bar{X}_{n}\right)\right] \text {. }
$$

A specific case of decomposable operation is a representable interval-valued aggregation w.r.t. the $\leq$-order.

\section{1 $\langle L([0,1]), \leq\rangle$-representable fuzzy connectives}

An interval-valued fuzzy connective may be considered as an interval representation of a fuzzy connective [4]. This generalisation fits the fuzzy principle and, the interval-valued degree of membership may be 
regarded as an approximation of the exact degree. See, the next studies of aggregations on $L([0,1])$, which are important in decision making applications.

Definition 8. A function $\mathbb{M}: L([0,1])^{n} \rightarrow L([0,1])$ is an interval-valued aggregation (IvA) function w.r.t. the partial $\leq$-order, if $\forall i \in \mathbb{N}_{n}$ and $\forall X_{i}, Y_{i} \in L([0,1])$, $\mathbb{M} 1: X_{i} \leq Y_{i} \Rightarrow \mathbb{M}\left(X_{1}, \ldots, X_{n}\right) \leq \mathbb{M}\left(Y_{1}, \ldots, Y_{n}\right)$; $\mathbb{M} 2: \mathbb{M}(\mathbf{0}, \ldots, \mathbf{0})=0$ and $\mathbb{M}(\mathbf{1}, \ldots, \mathbf{1})=1$.

Let $\mathbb{M}: L([0,1])^{n} \rightarrow L([0,1])$ be an IvA. $\mathbb{M}$ is $\langle L([0,1]), \leq\rangle$-representable if, there exist aggregation functions $M_{1}, M_{2}:[0,1]^{n} \rightarrow[0,1]$ such that $A_{1} \leq A_{2}$ and, $\forall X_{1}, \ldots, X_{n} \in L([0,1])$, the following holds:

$$
\mathbb{M}\left(X_{1}, \ldots, X_{n}\right)=\left[M_{1}\left(\underline{X}_{1}, \ldots, \underline{X}_{n}\right), M_{2}\left(\bar{X}_{1}, \ldots, \bar{X}_{n}\right)\right] .
$$

Notice that, a t-(co)norm is a function $T(S):[0,1]^{2} \rightarrow$ $[0,1]$ which is commutative, associative, monotonic and has $1(0)$ as neutral element. We consider an extension of the notion of the $\mathrm{t}-(\mathrm{co})$ norm to $\mathrm{L}([0,1])$ as it was introduced in [4].

Definition 9. A function $\mathbb{T}(\mathbb{S}): L([0,1])^{2} \rightarrow L([0,1])$ is an interval-valued t-norm (t-conorm), shortened by IvT (IvS), if it is commutative, associative, monotonic w.r.t. the product order, and has $\mathbf{1}(\mathbf{0})$ as the neutral element.

Example 5. See the usual $t$-(co)norms:

$$
\begin{aligned}
& T_{M}(x, y)=\min (x, y) \quad S_{M}(x, y)=\max (x, y) \\
& T_{P}(x, y)=x \cdot y \quad S_{P}(x, y)=x+y-x y \\
& T_{L K}(x, y)=\max (0, x+y-1) \quad S_{L K}(x, y)=\min (x+y, 1) \\
& T_{D}(x, y)=\left\{\begin{array}{l}
0, \text { if } x, y \in[0,1) \\
\min (x, y), \text { otherwise. }
\end{array} S_{D}(x, y)=\left\{\begin{array}{l}
1, \text { if } x, y \in(0,1] \\
\max (x, y), \\
\text { otherwise. }
\end{array}\right.\right.
\end{aligned}
$$

And, the Schweizer-Sklar t-conorm:

$$
S_{S S}(x, y)=1-\sqrt{\max \left((1-x)^{2}+(1-y)^{2}-1,0\right)}
$$

By Eq. (7), the functions $\mathbb{T}_{T, T^{\prime}}\left(\mathbb{S}_{S, S^{\prime}}\right): L([0,1])^{2} \rightarrow$ $L([0,1])$, are named as $\langle L([0,1]), \leq\rangle$-representable t(co)norm given by

$$
\begin{aligned}
\mathbb{T}_{T, T^{\prime}}(X, Y) & =\left[T(\underline{X}, \underline{Y}), T^{\prime}(\bar{X}, \bar{Y})\right], \\
\mathbb{S}_{S, S^{\prime}}(X, Y) & =\left[S(\underline{X}, \underline{Y}), S^{\prime}(\bar{X}, \bar{Y})\right],
\end{aligned}
$$

iff $T(x, y) \leq T^{\prime}(x, y) \quad\left(S(x, y) \leq S^{\prime}(x, y)\right), \forall x, y \in[0,1]$. And, for $\langle L([0,1]), \leq\rangle$-representable $\mathrm{t}$-(co)norm we have $T_{T, T^{\prime}}([0,1],[0,1])=[0,1]\left(S_{S, S^{\prime}}([0,1],[0,1])=\right.$ $[0,1])$. And, if $T=T^{\prime}\left(S=S^{\prime}\right)$, we get $\mathbb{T}_{T}\left(\mathbb{S}_{S}\right)$. By [4], $\mathbb{T}_{T}=\widehat{T}\left(\mathbb{S}_{S}=\widehat{S}\right)$ is characterised as $\widehat{T}(\widehat{S})$.

$\langle L([0,1]), \leq\rangle$-representable t-(co)norms based on usual $\mathrm{t}$-(co)norms from Example 5 are presented in Table 1.

In addition, an IvT (IvS) is representable iff it is monotonic w.r.t. the partial $\subseteq$-order [6]. However, not all $\mathrm{t}$-(co)norms on $L([0,1])$ are t-representable.
Table 1: $\langle L([0,1]), \leq\rangle$-representable t-(co)norms

$$
\begin{aligned}
& \mathbb{T}_{M}(X, Y)=[\min (\underline{X}, \underline{Y}), \min (\bar{X}, \bar{Y})] \\
& \mathbb{T}_{P}(X, Y)=[\underline{X Y}, \overline{X Y}] \\
& \mathbb{T}_{L K}^{*}(X, Y)=[\max (0, \underline{X}+\underline{Y}-1), \max (0, \bar{X}+\bar{Y}-1)] \\
& \mathbb{T}_{D}(X, Y)=\left\{\begin{array}{c}
\min (X, Y), \text { if } \max (X, Y)=\mathbf{1}, \\
{[0, \min (\bar{X}, \bar{Y})], \text { if } \max (\bar{X}, \bar{Y})=1 \text { and }} \\
\max (\underline{X}, \underline{Y}) \neq 1, \\
\mathbf{0}, \text { otherwise. }
\end{array}\right. \\
& \mathbb{S}_{M}(X, Y)=[\max (\underline{X}, \underline{Y}), \max (\bar{X}, \bar{Y})] \\
& \mathbb{S}_{P}(X, Y)=[\underline{X}+\underline{Y}-\underline{X} Y, \bar{X}+\bar{Y}-\bar{X} Y] \\
& \mathbb{S}_{L K}(X, Y)=[\min (\underline{X}+\underline{Y}, 1), \min (\bar{X}+\bar{Y}, 1)] \\
& \mathbb{S}_{D}(X, Y)=\left\{\begin{array}{lr}
\max (X, Y), \text { if } \min (X, Y)=\mathbf{0}, \\
{[\max (\underline{X}, \underline{Y}), 1], \text { if } \min (\underline{X}, \underline{Y})=0 \text { and }} \\
\mathbf{1}, \text { otherwise. }
\end{array}\right.
\end{aligned}
$$

Let $T(S)$ be a t-(co)norm on $L([0,1])$. The functions $\mathbb{T}_{S}^{\star}\left(\mathbb{S}_{T}^{\star}\right): L([0,1])^{2} \rightarrow L([0,1])$ given by

$$
\begin{array}{r}
\mathbb{T}_{T}^{\star}(X, Y)=[T(\underline{X}, \underline{Y}), \max (T(\underline{X}, \bar{Y}), T(\bar{X}, \underline{Y}))] ; \\
\mathbb{S}_{S}^{\star}(X, Y)=[\min (S(\underline{X}, \bar{Y}), S(\bar{X}, \underline{Y})), S(\bar{X}, \bar{Y})] .
\end{array}
$$

are called as pseudo $\langle L([0,1]), \leq\rangle$-representable t(co)norm, meaning that $\mathbb{T}_{T}^{\star}\left(\mathbb{S}_{S}^{\star}\right)$ is not t-representable. And, for $\mathbb{T}_{S}^{\star}\left(\mathbb{S}_{T}^{\star}\right)$, we have that $\mathbb{T}_{T}^{\star}([0,1],[0,1])=\mathbf{0}$ $\left(\mathbb{S}_{S}^{\star}([0,1],[0,1])=\mathbf{1}\right)$.

See, Table 2 , illustrating pseudo $\langle L([0,1]), \leq\rangle-$ representable $\mathrm{t}-(\mathrm{co})$ norms related to Example 5.

Table 2: Pseudo $\langle L([0,1]), \leq\rangle$-representability

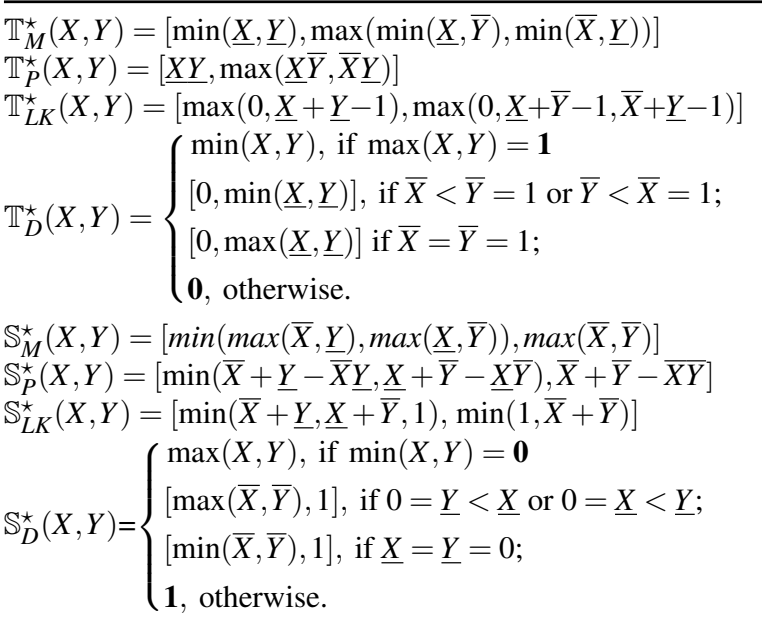

Now, let $T(S)$ be a t-(co)norm on $L([0,1])$. Based on results presented in [6], the function $\mathbb{T}_{T}^{\prime}\left(\mathbb{S}_{S}^{\prime}\right)(X, Y): L([0,1])^{2} \rightarrow L([0,1])$, given by

$$
\begin{aligned}
\mathbb{T}_{T}^{\prime}(X, Y) & =[\min (T(\underline{X}, \bar{Y}), T(\bar{X}, \underline{Y})), T(\bar{X}, \bar{Y})] ; \\
\mathbb{S}_{S}^{\prime}(X, Y) & =[S(\underline{X}, \underline{Y}), \max (S(\underline{X}, \bar{Y}), S(\bar{X}, \underline{Y}))],
\end{aligned}
$$


Table 3: Illustrating the class $\mathbb{T}_{T}^{\prime}\left(\mathbb{S}_{S}^{\prime}\right)$.

$\mathbb{T}_{M}^{\prime}(X, Y)=[\min (\min (\underline{X}, \bar{Y}), \min (\bar{X}, \underline{Y})), \min (\bar{X}, \bar{Y})] ;$ $\mathbb{T}_{P}^{\prime}(X, Y)=[\min (\underline{X} \bar{Y}, \bar{X} \underline{Y}), \overline{X Y}]$

$\mathbb{T}_{L K}^{\prime}(X, Y)=[\min (\max (0, \underline{X}+\bar{Y}-1)$, $\max (0, \bar{X}+\underline{Y}-1)), \max (0, \bar{X}+\bar{Y}-1)] ;$

$\mathbb{T}_{D}^{\prime}(X, Y)=\left\{\begin{array}{l}\min (X, Y), \text { if } \max (\bar{X}, \bar{Y})=1 ; \\ {[0, \bar{Y}], \text { if } \bar{Y}<\bar{X}=1 ;} \\ {[0, \bar{X}], \text { if } \bar{X}<\bar{Y}=1 ;} \\ \text { 0, otherwise. }\end{array}\right.$

$\mathbb{S}_{M}^{\prime}(X, Y)=[\max (\underline{X}, \underline{Y}), \max (\max (\underline{X}, \bar{Y}), \max (\bar{X}, \underline{Y}))] ;$

$\mathbb{S}_{P}^{\prime}(X, Y)=[\underline{X}+\underline{Y}-\underline{X} Y, \max (\underline{X}+\bar{Y}-\underline{X} \bar{Y}, \bar{X}+\underline{Y}-\bar{X} \underline{Y})]$;

$\mathbb{S}_{L K}^{\prime}(X, Y)=[\min (\underline{X}+\underline{Y}, 1)$ $\max (\min (\underline{X}+\bar{Y}, 1), \min (\bar{X}+\underline{Y}, 1))]$;

$\mathbb{S}_{D}^{\prime}(X, Y)=\left\{\begin{array}{l}\max (X, Y), \text { if } \min (\underline{X}, \underline{Y})=0 ; \\ {[\underline{Y}, 1], \text { if } \underline{Y}>\underline{X}=0 ;} \\ {[\underline{X}, 1], \text { if } \underline{X}>\underline{Y}=0 ;} \\ \mathbf{1}, \text { otherwise. }\end{array}\right.$

is not a t-representable $\mathrm{t}$-(co)norm, if $T \neq \min (S \neq$ $\max )$. See Table 3 for illustrations.

Definition 10. [4, Def. 4.1] A function $\mathbb{N}: L([0,1]) \rightarrow$ $L([0,1])$ is an interval-valued fuzzy negation (IvN) if, for all $X, Y \in L([0,1])$, it holds that:

$\mathbb{N} 1: \mathbb{N}(\mathbf{0})=\mathbf{1}$ and $\mathbb{N}(\mathbf{1})=\mathbf{0}$;

$\mathbb{N} 2 a$ : If $X \geq Y$, then $\mathbb{N}(X) \leq \mathbb{N}(Y)$;

$\mathbb{N} 2 b:$ If $X \subseteq Y$, then $\mathbb{N}(X) \subseteq \mathbb{N}(Y)$.

An IvN $\mathbb{N}$ is strong if it satisfies the involution property: $\mathbb{N} 3: \mathbb{N}(\mathbb{N}(X))=X, \forall X \in L([0,1])$.

According to $[4,7]$, we can construct a strong IvN $\mathbb{N}$ from a strong fuzzy negation $\mathrm{N}$, which is given by $\mathbb{N}(X)=[N(\bar{X}), N(\underline{X})]$. See, illustrations in Table 4 .

Table 4: Interval-Valued Fuzzy Negations

\begin{tabular}{|c|c|}
\hline$N_{C}(x)=1-x$ & $\mathbb{N}_{C}(X)=[1-\bar{X}, 1-\underline{X}]$ \\
\hline$N_{K}(x)=1-x^{2}$ & $\mathbb{N}_{K}(X)=\left[1-\bar{X}^{2}, 1-\underline{X}^{2}\right]$ \\
\hline$N_{R}(x)=1-\sqrt{x}$ & $\mathbb{N}_{R}(X)=[1-\sqrt{\bar{X}}, 1-\sqrt{\underline{X}}]$ \\
\hline$N_{D 1}(x)=\left\{\begin{array}{l}1, \text { if } x=0 \\
0, \text { otherwise }\end{array}\right.$ & $\mathbb{N}_{D 1}(X)=\left\{\begin{array}{l}\mathbf{1}, \text { if } X=\mathbf{0} \\
{[0,1], \text { if } 0 \in X} \\
\mathbf{0}, \text { otherwise }\end{array}\right.$ \\
\hline$N_{D 2}(x)=\left\{\begin{array}{l}1, \text { if } x<1 \\
0, \text { otherwise }\end{array}\right.$ & $\mathbb{N}_{D 2}(X)=\left\{\begin{array}{l}\mathbf{0}, \text { if } X=\mathbf{1} \\
{[0,1], \text { if } 1 \in X} \\
\mathbf{1}, \text { otherwise }\end{array}\right.$ \\
\hline
\end{tabular}

According to the idea that values in interval mathematics are identified with degenerate intervals, the minimal properties of fuzzy implications can be naturally extended for the interval-valued approach. Thus, by [13], for all $X, Y, Z \in L([0,1])$, an interval-valued function $\mathbb{I}: L([0,1])^{2} \rightarrow L([0,1])$ is an interval-valued fuzzy implication (IvI), if the next conditions hold:

$\mathbb{I} 1: \mathbb{I}(\mathbf{1}, \mathbf{1})=\mathbb{I}(\mathbf{0}, \mathbf{0})=\mathbb{I}(\mathbf{0}, \mathbf{1})=\mathbf{1}$, and $\mathbb{I}(\mathbf{1}, \mathbf{0})=\mathbf{0}$;

$\mathbb{I} 2:$ If $X \leq Z$, then $\mathbb{I}(X, Y) \geq \mathbb{I}(Z, Y)$;

$\mathbb{I} 3:$ If $Y \leq Z$, then $\mathbb{I}(X, Y) \leq \mathbb{I}(X, Z)$.

It is always possible to obtain, canonically, an IvI from any fuzzy implication, which also meets the optimality property and preserves the same properties satisfied by the fuzzy implication. Now, as a particular case of Eq.(5), the best interval representation $\widehat{I}$ of a fuzzy implication $I$, is shown as an inclusion-monotonic function in both arguments.

Proposition 11. [2, Prop. 16] If $I$ is a fuzzy implication then $\widehat{I}$ is an IvI.

Proposition 12. [2, Prop. 21] An implication $I:[0,1]^{2} \rightarrow[0,1]$ satisfies properties $\boldsymbol{I} \boldsymbol{I}$ and $\boldsymbol{I} \boldsymbol{2}$ iff $\widehat{I}$ can be expressed as: $\widehat{I}(X, Y)=[I(\bar{X}, \underline{Y}), I(\underline{X}, \bar{Y})]$.

An interval-valued fuzzy implication $\mathbb{I}_{\mathbb{S}, \mathbb{N}, \mathbb{T}}$ is an interval-valued QL-implication if there is an interval-valued t-conorm $\mathbb{S}$, an IvN $\mathbb{N}$ and an intervalvalued t-norm $\mathbb{T}$, such as

$$
\mathbb{I}_{\mathbb{S}, \mathbb{N}, \mathbb{T}}(X, Y)=\mathbb{S}(\mathbb{N}(X), \mathbb{T}(X, Y)) .
$$

Theorem 13. [13, Theo. 4] Let $S$ be a t-conorm, $N$ be a fuzzy negation and $T$ be a t-norm. Then, the following holds: $\mathbb{I}_{\widehat{S}, \widehat{N}, \widehat{T}}=\widehat{I_{S, N, T}}$.

Corollary 2. [13, Corollary. 1] II is an interval-valued $Q L$-implication iff there exist an QL-implication I such that $\mathbb{I}=\widehat{I}$.

Proposition 14. [13, Proposition 6] Let II be an IvI. If $\mathbb{I}$ is an interval-valued QL-implication, then the properties $\mathbb{I} 2$ and $\mathbb{I} 3$ are met.

Extensions of QL-implications [6] are given as:

$$
\begin{aligned}
\mathbb{I}_{\mathbb{S}_{L K}, \mathbb{N}_{C}, \mathbb{T}_{M}}(X, Y) & =\mathbb{I}_{L K}(X, Y) ; \\
\mathbb{I}_{\mathbb{S}_{L K}, \mathbb{N}_{C}, \mathbb{T}_{P}}(X, Y) & =\mathbb{I}_{R C}(X, Y) ; \\
\mathbb{I}_{\mathbb{S}_{D}, \mathbb{N}_{C}, \mathbb{T}_{P}}(X, Y) & =\mathbb{I}_{D P}(X, Y) ; \\
\mathbb{I}_{\mathbb{S}_{L K}, \mathbb{N}_{C}, \mathbb{T}_{L K}}(X, Y) & =\mathbb{I}_{K D}(X, Y) .
\end{aligned}
$$

See in Table 5, the representations of such operators. Moreover, some implications are exclusively QLimplications (meaning they do not belong to other classes, like S-implications or R-implications). Some examples from [6] are reported below.

$$
\begin{aligned}
\mathbb{I}_{\mathbb{S}_{L K}, \mathbb{N}_{K}, \mathbb{T}_{P}}(X, Y) & =\mathbb{I}_{K P}(X, Y) ; \\
\mathbb{I}_{\mathbb{S}_{S S}, \mathbb{N}_{C}, \mathbb{T}_{P}}(X, Y) & =\mathbb{I}_{P C}(X, Y) ; \\
\mathbb{I}_{\mathbb{S}_{S S}, \mathbb{N}_{R}, \mathbb{T}_{P}}(X, Y) & =\mathbb{I}_{P R}(X, Y) .
\end{aligned}
$$

Their representations were also considered in Table 6 .

\section{Representability in $\left\langle L\left([0,1], \preceq_{A}\right\rangle\right.$}

The concept of $\left\langle L([0,1]), \preceq_{A}\right\rangle$-connectives is now presented as a more general setting for an arbitrary admissible $\left\langle L([0,1]), \preceq_{A}\right\rangle$-order. 
Table 5: Examples of QL-Implications.

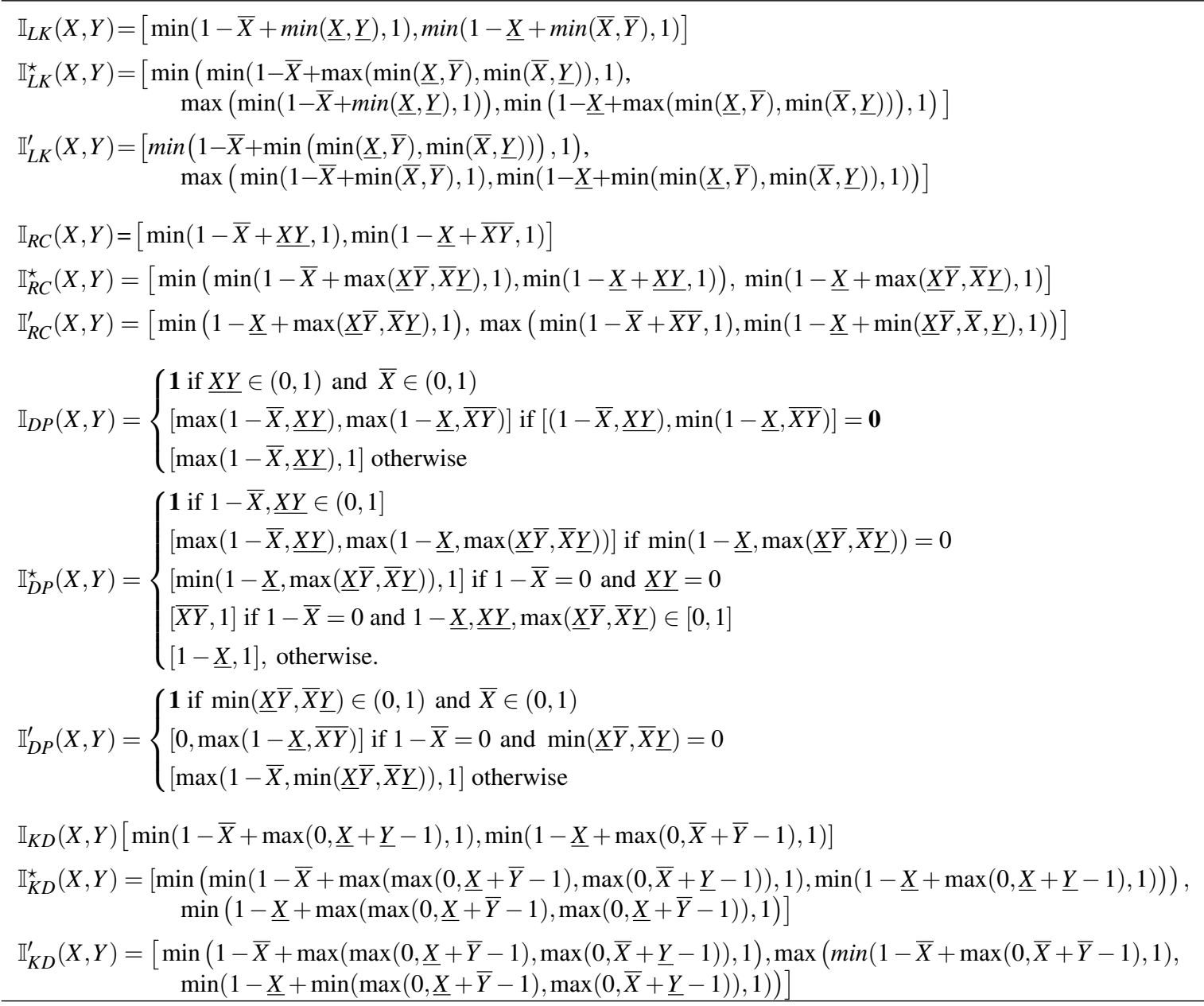

Proposition 15. Let $M:[0,1]^{n} \rightarrow[0,1]$ be (a strictly increasing) an aggregation function, let $A$ be a function verifying the conditions of Theorem 2 . The function $\mathbb{M}^{A}: L([0,1])^{n} \rightarrow L([0,1])$, given as

$\mathbb{M}^{A}\left(X_{1}, \ldots, X_{n}\right)=A^{(-1)}\left(M\left(A\left(X_{1}\right), \ldots, A\left(X_{n}\right)\right)\right)$

is (a strictly increasing) an IvA function related to the admissible $\preceq_{A}$-order, in the sense of Definition 8 .

Proof. Trivially, the boundary conditions are verified: $\mathbb{M}^{A}(\mathbf{0}, \ldots, \mathbf{0})=A^{(-1)}(M(A(\mathbf{0}), \ldots, A(\mathbf{0})))=$ $A^{(-1)}(M(0, \ldots, 0))=\mathbf{0}$ and, besides, $\mathbb{M}^{A}(\mathbf{1}, \ldots, \mathbf{1})=$ $A_{1}^{(-1)}(M(A(\mathbf{1}), \ldots, A(\mathbf{1})))=A_{1}^{(-1)}(M(1, \ldots, 1))=\mathbf{1}$. Let $X_{1}, \ldots, X_{n}, Y \in L([0,1])$, such that $X_{i} \preceq_{A} Y$, for some $i \in \mathbb{N}_{n}$. Then, $A\left(X_{i}\right) \leq A(Y)$ and because $M$ is strictly increasing, $M\left(A\left(X_{1}\right), \ldots, A\left(X_{n}\right)\right) \leq$ $M\left(A\left(X_{1}\right), \ldots, A\left(X_{i-1}\right), A(Y), A\left(X_{i+1}\right), \ldots, A\left(X_{n}\right)\right)$ and so, by Lemma $1, \mathbb{M}^{A}\left(X_{1}, \ldots, X_{n}\right) \preceq_{A} \mathbb{M}^{A}\left(X_{1}, \ldots, X_{i-1}\right.$, $\left.Y, X_{i+1}, \ldots, X_{n}\right)$, In addition, since $A$ is injective, when $M$ is strictly increasing, $M_{A}$ is also strictly increasing. Therefore, Proposition 15 is verified.
Prop. 15 also holds for $\mathbf{A}$ and $\mathbf{A}^{(-1)}$ given in Def. 3,5. Table 7 shows the extensions of t-norms from Table 1 .

Theorem 16. Let $N:[0,1] \rightarrow[0,1]$ be a strictly decreasing fuzzy negation, $A: L([0,1]) \rightarrow[0,1]$ and $A$ and $A^{(-1)}$ be functions verifying the conditions of Theorem 2, as required in Lemma 1. The function $\mathbb{N}^{A}: L([0,1]) \rightarrow L([0,1])$ defined by

$$
\mathbb{N}^{A}(X)=A^{(-1)}(N(A(X))) .
$$

is an $\left\langle L([0,1]), \preceq_{\mathbf{A}}\right\rangle$-negation, which will be called $a$ representable $\left\langle L([0,1]), \preceq_{A}\right\rangle$-negation.

Proof. Trivially, $\quad \mathbb{N}^{\mathbf{A}}(\mathbf{0})=\mathbf{1}$ and $\mathbb{N}^{\mathbf{A}}(\mathbf{1})=\mathbf{0}$. When $X \preceq_{\mathbf{A}} Y$, since $N$ is strictly decreasing and Lemma 1, $A(X)<A(Y) \Leftrightarrow N(A(Y))<N(A(X))) \Leftrightarrow$ $A^{(-1)}(N(A(Y)))<A^{(-1)}(N(A(X))) \Leftrightarrow \mathbb{N}^{A}(Y) \preceq_{A}$ $\mathbb{N}^{A}(X)$. So, $\mathbb{N}^{A}$ is an $\left\langle L([0,1]), \preceq_{A}\right\rangle$-negation.

An $\left.\langle L([0,1]]), \preceq_{\mathbf{A}}\right\rangle$-negation $\mathbb{N}: L([0,1]) \rightarrow L([0,1])$ is a function verifying $(\mathbb{N} 1)$ and the following property: $\left(\mathbb{N}_{\mathbf{A}} 2\right)$ : If $X \preceq_{\mathbf{A}} Y, \mathbb{N}(Y) \preceq_{\mathbf{A}} \mathbb{N}(X), \forall X, Y \in L([0,1])$. 
Table 6: Illustrating (exclusive) QL-Implications on $\langle L([0,1]), \leq\rangle$.

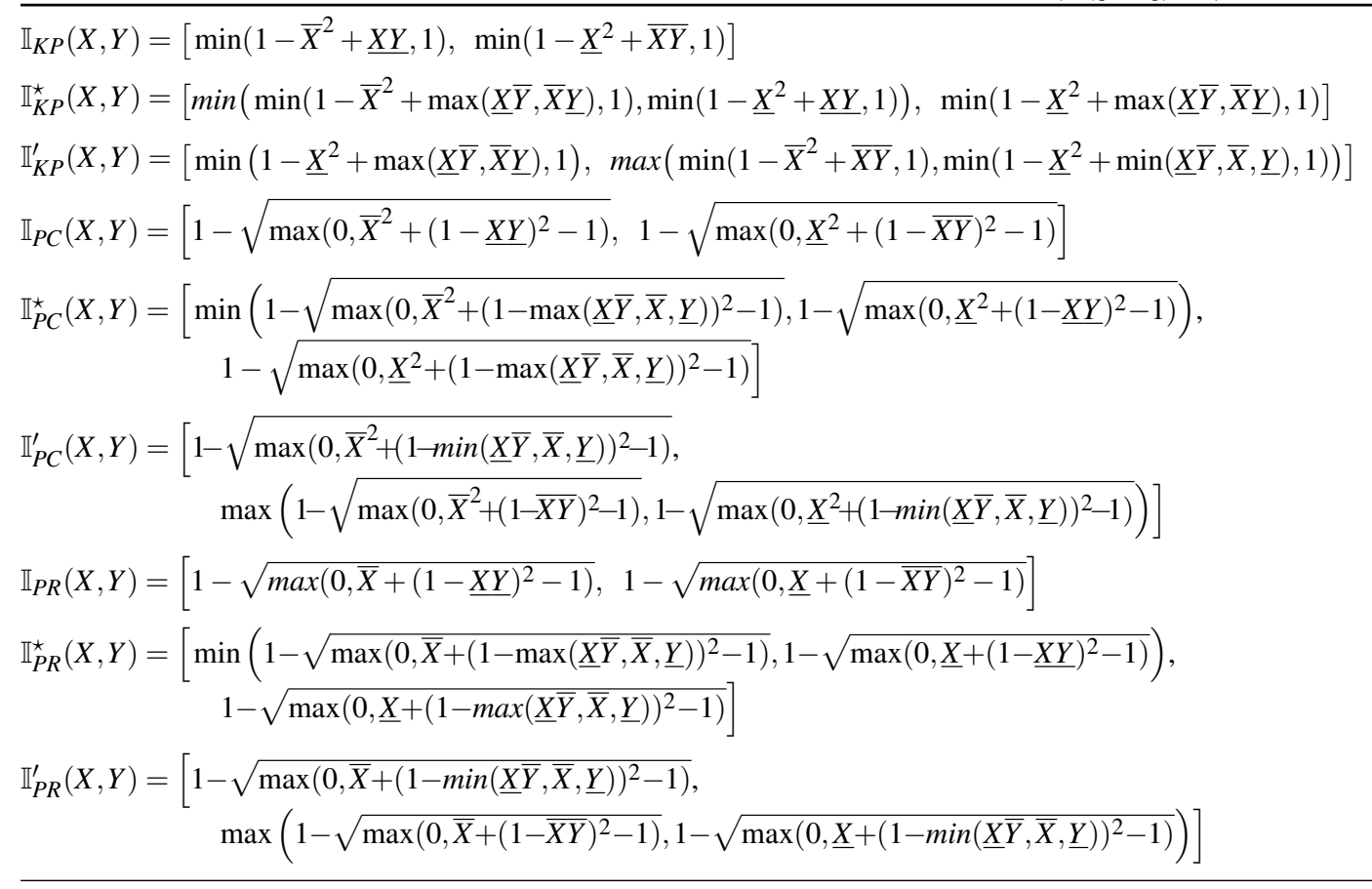

Table 7: Representable t-(co)norms on $\left\langle L([0,1]), \preceq_{\mathbf{A}}\right\rangle$.

$$
\begin{aligned}
& \mathbb{T}_{M}^{\mathbf{A}}(X, Y)=\mathbf{A}^{(-1)}(\min (\mathbf{A}(X), \mathbf{A}(Y))) \\
& \mathbb{T}_{P}^{\mathbf{A}}(X, Y)=\mathbf{A}^{(-1)}(\mathbf{A}(X) \cdot \mathbf{A}(Y)) \\
& \mathbb{T}_{L K}^{\mathbf{A}}(X, Y)=\mathbf{A}^{(-1)}(\max (0, \mathbf{A}(X)+\mathbf{A}(Y)-1)) \\
& \mathbb{T}_{D}^{\mathbf{A}}(X, Y)=\left\{\begin{array}{l}
\mathbf{0}, \text { if } \mathbf{A}(X), \mathbf{A}(Y) \in[0,1) \\
\mathbf{A}^{(-1)}(\min (\mathbf{A}(X), \mathbf{A}(Y))), \text { otherwise }
\end{array}\right. \\
& \mathbb{S}_{M}^{\mathbf{A}}(X, Y)=\mathbf{A}^{(-1)}(\max (\mathbf{A}(X), \mathbf{A}(Y))) \\
& \mathbb{S}_{P}^{\mathbf{A}}(X, Y)=\mathbf{A}^{(-1)}(\mathbf{A}(X)+\mathbf{A}(Y)-\mathbf{A}(X) \cdot \mathbf{A}(Y)) \\
& \mathbb{S}_{L K}^{\mathbf{A}}(X, Y)=\mathbf{A}^{(-1)}(\min (\mathbf{A}(X)+\mathbf{A}(Y), 1)) \\
& \mathbb{S}_{D}^{\mathbf{A}}(X, Y)=\left\{\begin{array}{l}
\mathbf{1}, \text { if } \mathbf{A}(X), \mathbf{A}(Y) \in(0,1] \\
\mathbf{A}^{(-1)}(\max (\mathbf{A}(X), \mathbf{A}(Y))), \text { otherwise }
\end{array}\right.
\end{aligned}
$$

Corollary 3. Whenever $N:[0,1] \rightarrow[0,1]$ is a strong fuzzy negation, $\mathbb{N}^{A}$ verifies $\mathbb{N}^{A}\left(\mathbb{N}^{A}(X)\right) \succeq_{A} X$.

Proof. By Lemma1, we have that $\mathbb{N}^{A}\left(\mathbb{N}^{A}(X)\right) \succeq_{A}$ $A^{(-1)}\left(N^{2}(A(X))\right)$. Therefore, $\mathbb{N}^{A}\left(\mathbb{N}^{A}(X)\right) \succeq_{A} X$.

Example 6. See, the $\left\langle L([0,1]), \preceq_{\mathbf{A}}\right\rangle$-representable negation generated by the standard negation $N_{C}$ :

$$
\mathbb{N}_{C}^{\mathbf{A}}(X)=\mathbf{A}^{(-1)}\left(N_{C}(\mathbf{A}(X))\right), \forall X \in L([0,1]) .
$$

By Example 2, $\quad \mathbb{N}_{C}^{\mathbf{A}}(X)=\mathbf{A}^{(-1)} N_{C}(0.1858)=$ $\mathbf{A}^{(-1)}(0.8142)=[0.8,0.8]$ and $\mathbb{N}_{C} \mathbf{A}(Y)=[0.8,0.8]$. Here $X \preceq_{\mathbf{A}} Y, \mathbb{N}_{C}^{\mathbf{A}}(Y) \preceq_{\mathbf{A}} \mathbb{N}_{C}^{\mathbf{A}}(X)$, hence, $\mathbb{N}_{S}^{\mathbf{A}}$ is not $a$ strong negation.
An $\left\langle L([0,1]), \preceq_{\mathbf{A}}\right\rangle$-implication $\mathbb{I}: L([0,1])^{2} \rightarrow L([0,1])$ is a function verifying $\mathbb{I} 1$ and the following conditions: $\mathbb{I}_{\mathbf{A}} 2$ : If $X \preceq_{\mathbf{A}} Y$, then $\mathbb{I}(Y, Z) \preceq_{\mathbf{A}} \mathbb{I}(X, Z)$; $\mathbb{I}_{\mathbf{A}} 3$ : If $Y \preceq_{\mathbf{A}} Z$, then $\mathbb{I}(X, Y) \preceq_{\mathbf{A}} \mathbb{I}(X, Z)$.

Theorem 17. Let $I:[0,1]^{2} \rightarrow[0,1]$ be a fuzzy implication, $\mathbf{A}: L([0,1]) \rightarrow[0,1]$ and $\mathbf{A}^{(-1)}:[0,1] \rightarrow L([0,1])$ be functions as in Def. 3 and 5, as required in Lemma 1 . The function $\mathbb{I}^{\mathbf{A}}: L([0,1])^{2} \rightarrow L([0,1])$ defined by

$$
\mathbb{I}^{\mathbf{A}}(X, Y)=\mathbf{A}^{(-1)}(I(\mathbf{A}(X), \mathbf{A}(Y))),
$$

is an $\left\langle L([0,1]), \preceq_{\mathbf{A}}\right\rangle$-implication, which will be called a representable $\left\langle L([0,1]), \preceq_{\mathbf{A}}\right\rangle$-implication.

Proof. Let $I:[0,1]^{2} \rightarrow[0,1]$ be a fuzzy implication. So, the boundary conditions are verified:

$\mathbb{I}^{\mathbf{A}}(\mathbf{0}, \mathbf{0})=\mathbf{A}^{(-1)}(I(\mathbf{A}(\mathbf{0}), \mathbf{A}(\mathbf{0})))=\mathbf{A}^{(-1)}(I(0,0))=\mathbf{1} ;$ $\mathbb{I}^{\mathbf{A}}(\mathbf{0}, \mathbf{1})=\mathbf{A}^{(-1)}(I(\mathbf{A}(\mathbf{0}), \mathbf{A}(\mathbf{1})))=\mathbf{A}^{(-1)}(I(0,1))=\mathbf{1} ;$ $\mathbb{I}^{\mathbf{A}}(\mathbf{1}, \mathbf{0})=\mathbf{A}^{(-1)}(I(\mathbf{A}(\mathbf{1}), \mathbf{A}(\mathbf{0})))=\mathbf{A}^{(-1)}(I(1,0))=\mathbf{0} ;$ $\mathbb{I}^{\mathbf{A}}(\mathbf{1}, \mathbf{1})=\mathbf{A}^{(-1)}(I(A(\mathbf{1}), A(\mathbf{1})))=\mathbf{A}^{(-1)}(I(1,1))=\mathbf{1}$. (II) If $X_{1} \preceq_{\mathbf{A}} X_{2}$, either $\mathbf{A}\left(X_{1}\right)<\mathbf{A}\left(X_{2}\right)$ or $\mathbf{A}\left(X_{1}\right)=\mathbf{A}\left(X_{2}\right)$. By $I 1$, we have that $I\left(\mathbf{A}\left(X_{1}\right), \mathbf{A}(Y)\right) \geq I\left(\mathbf{A}\left(X_{2}\right), \mathbf{A}(Y)\right)$. Now, based on Lemma 1 and Equation (1), $\mathbb{I}^{\mathbf{A}}\left(X_{1}, Y\right)=$ $\mathbf{A}^{(-1)}\left(I\left(\mathbf{A}\left(X_{1}\right), \mathbf{A}(Y)\right)\right) \succeq_{\mathbf{A}} \mathbf{A}^{(-1)}\left(I\left(\mathbf{A}\left(X_{2}\right), \mathbf{A}(Y)\right)\right)=$ $\mathbb{I}^{\mathbf{A}}\left(X_{2}, Y\right)$. Otherwise, if $\mathbf{A}\left(X_{1}\right)=\mathbf{A}\left(X_{2}\right)$, then $\mathbf{A}^{(-1)}\left(\mathbb{I}^{\mathbf{A}}\left(X_{1}, Y\right)\right)=\mathbf{A}^{(-1)}\left(\mathbb{I}^{\mathbf{A}}\left(X_{2}, Y\right)\right)$.

(I2) Analogously done.

See QL-implications on $\left\langle L([0,1]), \preceq_{\mathbf{A}}\right\rangle$ in Table 8 . Observe that $\left\langle L([0,1]), \preceq_{A}\right\rangle$-representable QL- 
implications are those based on QL-implications.

Table 8: $\langle L([0,1]) \preceq \mathbf{A}\rangle$-Representable QL-implications

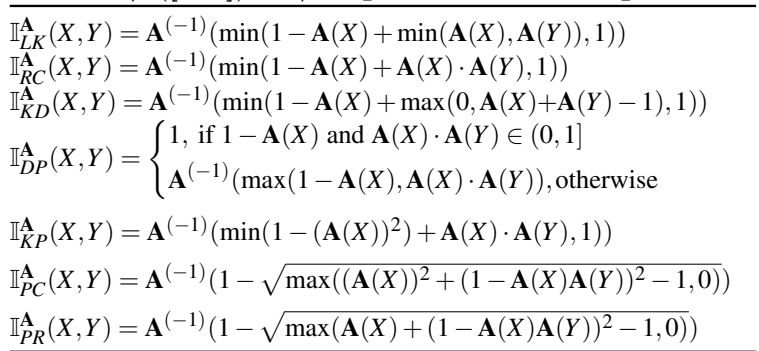

\section{Conclusion}

This work provided interval representation of QL-implications on $\mathrm{L}([0,1])$ endowed with partial $\langle L([0,1]), \leq\rangle$-order and the admissible $\left\langle L([0,1]), \preceq_{A}\right\rangle$ order, emphasizing properties of main classes of interval-valued fuzzy operators, namely IvT, IvS, IvN and IvI. An alternative approach to generate interval-valued connectives is introduced, allowing the study on the representability of these operators on $\left\langle L([0,1]), \preceq_{A}\right\rangle$ lattice. Further studies consider extensions of the $\preceq_{A}$-order on interval-valued intuitionistic fuzzy approach [1].

\section{Acknowledgement}

This work was supported by CNPq (309160/20197, 311429/2020-3) and FAPERGS (16/2551-00004889,17/2551-0001207-0,19/2551-0001279-9).

\section{References}

[1] K. Atanassov, G. Gargov, Interval valued intuitionistic fuzzy sets, Fuzzy Sets Syst 31 (3) (1989) 343-349.

[2] B. Bedregal, G. P. Dimuro, R. Santiago, R. Reiser, On interval fuzzy S-implications, Information Sciences 180 (8) (2010) 1373-1389.

[3] B. Bedregal, A. Takahashi, The best interval representations of t-norms and automorphisms, Fuzzy Sets Syst 157 (24) (2006) 3220-3230.

[4] B. Bedregal, A. Takahashi, Interval valued versions of t-conorms, fuzzy negations and fuzzy implications, in: IEEE Intl Conf on Fuzzy Systems, 2006, pp. 1981-1987.

[5] H. Bustince, J. Fernandez, A. Kolesárová, R. Mesiar, Generation of linear orders for intervals by means of aggregation functions, Fuzzy Sets Syst 220 (2013) $69-77$.
[6] G. Deschrijver, A representation of t-norms in interval-valued L-fuzzy set theory, Fuzzy Sets Syst 159 (13) (2008) 1597-1618.

[7] G. Deschrijver, C. Cornelis, Representability in interval-valued fuzzy set theory, Int Journal of Uncertainty, Fuzziness and Knowledge-Based Systems 15 (03) (2007) 345-361.

[8] T. Hickey, Q. Ju, M. H. Van Emden, Interval arithmetic: From principles to implementation, J. ACM 48 (5) (2001) 1038-1068.

[9] R. B. Kearfott, V. Kreinovich, Applications of interval computations, Vol. 3, Springer Science \& Business Media, 2013.

[10] M. Matzenauer, R. Reiser, H. S. Santos, B. R. C. Bedregal, H. Bustince, Strategies on admissible total orders over typical hesitant fuzzy implications applied to decision making problems, Int. J. Intell. Syst. 36 (5) (2021) 2144-2182.

[11] R. Moore, W. Lodwick, Interval analysis and fuzzy set theory, Fuzzy Sets Syst 135 (1) (2003) 5-9.

[12] B. Pekala, Uncertainty Data in Interval-Valued Fuzzy Set Theory: Properties, Algorithms and Applications, Vol. 367, Springer, 2018.

[13] R. H. S. Reiser, G. P. Dimuro, B. R. C. Bedregal, R. H. N. Santiago, Interval valued ql-implications, in: D. Leivant, R. J. G. B. de Queiroz (Eds.), Logic, Language, Information and Computation, 14th Int. Workshop, WoLLIC 2007, Rio de Janeiro, Brazil, 2007, Proc., Vol. 4576 of Lecture Notes in Computer Science, Springer, 2007, pp. 307-321.

[14] F. Santana, B. Bedregal, P. Viana, H. Bustince, On admissible orders over closed subintervals of [0,1], Fuzzy Sets Syst 399 (2020) 44-54.

[15] R. Santiago, B. Bedregal, B. Acióly, Formal aspects of correctness and optimality of interval computations, Formal Aspects of Computing 18 (2) (2006) 231-243.

[16] L. A. Zadeh, Fuzzy sets, Information and control 8 (3) (1965) 338-353.

[17] L. A. Zadeh, The concept of a linguistic variable and its application to approximate reasoning-I, Information Sciences 8 (3) (1975) 199-249.

[18] H. Zapata, H. Bustince, S. Montes, B. Bedregal, G. Dimuro, Z. Takác, M. Baczyński, J. Fernández, Interval-valued implications and intervalvalued strong equality index with admissible orders, Int J Approx Reason 88 (2017) 91-109. 